\title{
A Portable System with 0.1 ppm RMSE Resolution for 1-10 MHz Resonant MEMS Frequency Measurement
}

\author{
Laxmeesha Somappa, Adarsh G. Menon, Ajay K. Singh, Ashwin A. Seshia, Senior Member, IEEE and \\ Maryam Shojaei Baghini, Senior Member, IEEE
}

\begin{abstract}
This paper presents a portable and programmable frequency measurement system (PrO-FMS) with 0.1 ppm RMSE resolution over a measurement time interval of $33.2 \mathrm{~s}$ for bulkacoustic resonator applications. PrO-FMS has a feature of choosing a frequency estimation method and accordingly the sampling frequency, which is programmable. Five frequency estimation methods: Candan, Djukanovic, Prony, M-Pisarenko and zero crossing interpolation methods are reviewed for high resolution frequency measurement. PrO-FMS can be also reconfigured to select a particular resonance mode over a range of 1-10 MHz. Measurement results are provided for two different commercial quartz crystals and a microfabricated in-plane bulk acoustic resonator in two different modes of resonance. The results of these estimation methods are compared with a standard commercial tabletop frequency counter. Measurement results show that PrO-FMS can achieve a resolution of 0.1 ppm RMSE. For the same gate time, PrO-FMS provides better resolution than standard tabletop frequency counter. The presented work also provides a study on the behavior of the five different frequency estimation methods for short and long term measurements for quartz crystals and bulk acoustic resonator in two different modes.
\end{abstract}

Index Terms-MEMS, bulk-acoustic-wave, frequency estimation, portable electronics, high resolution.

\section{INTRODUCTION}

$\mathrm{Q}$ UARTZ crystals are used in many precise timing modules in today's electronic devices because of their high quality factor (Q). With the added advantage of miniaturization, silicon-based micro and nanomechanical resonators are being used to replace quartz crystals [1] as well as develop resonant sensors. MEMS gyroscopes are widely used in consumer electronics, automotive safety and robotics due to their small size and low power consumption. Bulk-acoustic-wave (BAW) gyroscopes have demonstrated higher sensitivity, larger dynamic range and better shock and vibration robustness compared to low-frequency tuning-fork-type gyroscopes [2], making them good candidates for such applications. BAW gyroscopes rely on the frequency-matching of high-Q gyroscopic resonant modes with frequencies in the $1-10 \mathrm{MHz}$ range to achieve high performance and high dynamic range [3]. Microfabricated in-plane bulk acoustic resonators in the frequency range 1-10 $\mathrm{MHz}$ have been configured as gravimetric sensors wherein the mass of surface-bound species is transduced as a shift in the resonant frequency for sub $100 \mathrm{~nm}$ particle mass measurement [4], [5]. Applications involving these bulk acoustic resonant devices rely highly on very precise measurements of the resonant frequency shift. This necessitate the need for a portable, highly precise frequency measurement systems for interfacing the bulk acoustic resonators with considerably small error in the presence of noise sources.

Tabletop frequency counters are most commonly used for resonant frequency/stability measurement of resonant devices. They use the principle of counting the number of pulses in a predetermined measurement interval (gate time) to estimate the frequency. Their counterpart, the reciprocal frequency counters [6], measure the input signal period over the gate time and reciprocates to obtain the frequency. The measurement accuracy of such systems is inversely proportional to the measurement interval and the accuracy of the internal oscillators. Consequently, oven-controlled crystal oscillators are mainly used to generate the time base for these systems. This makes the system bulky. Since these devices use an internal reference oscillator, drifts in the oscillator manifests itself as a measurement error.

As an alternative, time to digital converters (TDC) are used in portable, high resolution time/frequency measurement scenarios. [7] implements a portable TDC with high signal bandwidth. However, the TDC, encompassing an FPGA, achieves a resolution of not more than $30 \mathrm{ppm}$ even with a large measurement interval of $200 \mathrm{~ms}$. [8]-[12] discuss the implementation of pico-second resolution TDC's implemented on FPGA's. TDC's implemented on FPGA have shorter conversion time, better scalability and adaptability according to the needs of the application. However, for a given clock frequency or measurement time, these TDC's have a limited dynamic range which restricts the frequency tracking over a large bandwidth with very fine resolution. Also, the high reference clock requirements for these systems limit the measurement resolution.

Given a highly precise frequency measurement system such as a tabletop counter or a TDC, the measurement error still depends on the phase noise of the external oscillator circuit. Hence, there is a need for a system which integrates a tunable oscillator along with the high resolution frequency measurement system. The measurement system encompassing the oscillator has to be adaptable/programmable to support bulk acoustic resonator devices (exhibiting different phase noise behavior) over a wide frequency range of 1-10 $\mathrm{MHz}$.

In this paper, a portable, Programmable Frequency Measurement System (PrO-FMS) is proposed that can measure frequency with high resolution over a considerably small measurement interval. The conceptual block diagram is shown 


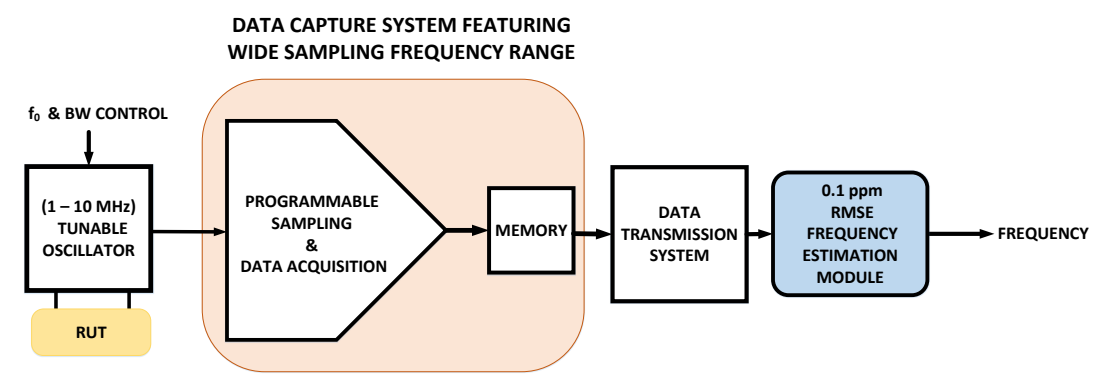

Fig. 1: The conceptual block diagram of PrO-FMS.

in Fig. 1.

The system contains a tunable oscillator loop which can be reconfigured based on the resonator under test (RUT) to support resonant devices in the frequency range 1-10 MHz. The output of the oscillator is fed to a data capture system, with a programmable sampling clock. This data is then transmitted to a central computing platform through $\mathrm{I} 2 \mathrm{C}$ or Bluetooth transmission. The computing platform then performs frequency estimation. In this paper, we review five different frequency estimation algorithms [13]-[17] which are suitable for high resolution and low measurement interval applications. Based on the application and the required resolution-measurement interval, any suitable algorithm can then be used for frequency estimation. In this system, the precise computation of the frequency is pushed into the computing platform rather than an on-board processor. With suitable choice of frequency measurement algorithms, the reference clock frequency can be substantially lowered as compared to a FPGA based TDC or a tabletop frequency counter. In the paper, the proposed system (PrO-FMS) is evaluated using a standard Agilent53230A reciprocal frequency counter. It is shown that PrOFMS is able to achieve resolution of $0.1 \mathrm{ppm}$ RMSE for a maximum measurement interval of $33.2 \mathrm{~s}$.

The paper is organized as follows. In Section II, the block level description of the system is presented. Section III describes the system-level implementation with the measurement protocol. Section IV discusses the absolute frequency calibration of PrO-FMS. The measurement results are provided for two different crystals and two closely spaced modes in section $\mathrm{V}$ followed by conclusions and future work in section VI.

\section{System Description AND System ImPLEMENTATION}

A tabletop frequency estimation system needs a standalone oscillator housing the resonator under test. The resonator output drives the sampling and data acquisition module which are part of the tabletop frequency estimation system. The frequency measurement is then performed on the tabletop system using a digital signal processor or an FPGA. This limits the nature of frequency estimation techniques that can be employed for precise frequency measurements over a small measurement time interval. Employing a central computing unit enables handling of a wide range of precise frequency estimation techniques over a small measurement time interval with added advantage of portability. The portable system can then be configured with very few changes for a target resolutionmeasurement interval requirement. Fig. 1 shows the conceptual block diagram of PrO-FMS. The oscillator, data acquisition, memory and the data transmission modules are part of a single circuit board. The data transmission module enables handling a wide range of precise frequency estimation techniques over a small measurement interval on the computing platform. PrOFMS has an added advantage of flexible integration with a sensor network.

\section{A. System Description}

Pro-FMS is a high resolution frequency measurement system which constitutes the following functional blocks: the wide range tunable (1-10 MHz) oscillator [18], programmabledata capture supporting wide sampling frequency range, data transmission and very high accuracy frequency estimation module. The oscillator circuit consists of a trans-impedance amplifier (TIA), multi feedback (MFB) bandpass filter and a comparator all operating at $3.3 \mathrm{~V}$ supply voltage. The amplitude information from the oscillator circuit is sampled and digitized by the data capture module. The data capture module has a an ADC and an onboard programmable clock. The onboard microcontroller programs the clock during startup. The sampled data is then transmitted to the frequency estimation module by the data capture system. The onboard microcontroller generates the control signals required for the data transfer to the frequency estimation module. The high accuracy frequency estimation module then estimates the frequency based on a suitable algorithm. In this work, five different estimation algorithms [13]-[17] are explored.

\section{B. System Implementation}

The proposed PrO-FMS is shown in Fig. 2. The system works on a 3.3V supply. The PrO-FMS has a tunable oscillator, programmable data capture, an on-board micro controller for synchronizing the data capture and the data transmission modules. The detailed description of each of the functional blocks will be provided in the subsequent subsections.

1) Oscillator: The oscillator circuit consisting of a transimpedance amplifier (TIA), MFB bandpass filter, comparator was designed and developed (Fig. 2). The RUT will be embedded in the feedback of the oscillator. The MFB band pass filter allows for tuning the oscillator loop for various RUT. The TIA converts the RUT output current to corresponding voltage. The TIA along with the bandpass filter provides gain tuning to obtain the required signal swing at the input of the ADC. Increasing the TIA gain increases the signal swing and hence the non-linearity in the loop. To cover a wide range of resonator frequencies (1-10 MHz), CMOS OpAmp OPA355 


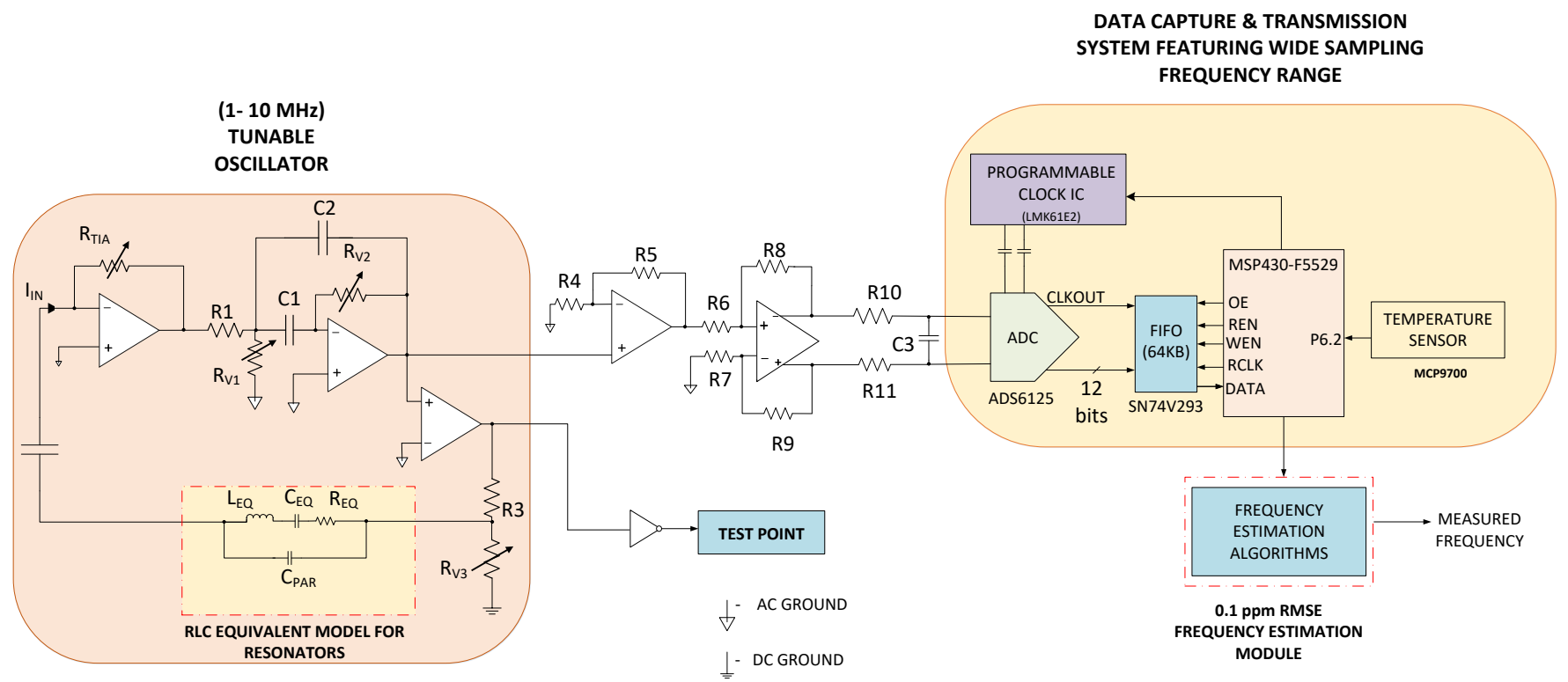

Fig. 2: The proposed 3.3V Programmable Frequency Measurement System (PrO-FMS).

(from Texas Instruments) having a wide bandwidth and very low noise of $5.8 \mathrm{nV} / \sqrt{\mathrm{Hz}}$ is used to make a low noise TIA implementation. Output of the TIA drives the MFB bandpass filter, which selects the frequency of oscillation. Assuming $C_{1}$ $=C_{2}=\mathrm{C}$ in Fig. 2 and neglecting finite opamp gain and the input parasitic capacitance, the transfer function of the band pass filter is given by Eq. 1.

$$
\frac{V_{o}}{V_{i n}} \approx \frac{-s R_{v 2} C}{s^{2} R_{1} R_{v 2} C^{2}+2 s R_{1} C+\left(1+\frac{R_{1}}{R_{v 1}}\right)}
$$

The bandpass filter has one zero at dc and two complex poles. The values of the resistors and capacitors for any RUT are decided based on the design equations tabulated in the Table I. By varying $R_{v 1}$ and $R_{v 2}$ the bandwidth and center frequency for the bandpass filter can be adjusted. This ensures that the loop can be tuned for a wide range of resonant frequencies. The same operational amplifiers used for the trans-impedance amplifier is used to implement the bandpass filter because of its high bandwidth. The comparator feeds the actuation signal to the RUT. The drive voltage to the RUT is controlled by means of a potential divider at the output of the comparator. This provides another degree of freedom on the oscillator output swing - non linearity tradeoff. A high-speed comparator TLV 3501 is used due to the low propagation delay in the order of few nanoseconds. The bandpass filter output drives a voltage buffer followed by a single-ended to differential converter along with an anti-aliasing filter.

TABLE I: Circuit parameters of the band pass filter.

\begin{tabular}{|l|c|}
\hline \multicolumn{1}{|c|}{ Circuit parameters } & Values \\
\hline Centre frequency $\left(\omega_{o}\right)$ & $\frac{1}{C} * \sqrt{\frac{1}{R_{v 2}}\left(\frac{1}{R_{1}}+\frac{1}{R_{v 1}}\right)}$ \\
\hline Bandwidth & $\frac{2}{R_{v 2} C}$ \\
\hline Quality factor $(\mathbf{Q})$ & $0.5 * \sqrt{\frac{R_{v 2}}{R_{1}}+\frac{R_{v 2}}{R_{v 1}}}$ \\
\hline Gain $\left(@ \omega_{o}\right)$ & $\frac{V_{o}}{V_{i n}}=\frac{-R_{v 2}}{2 R_{1}}$ \\
\hline
\end{tabular}

2) Data Capture and Data Transmission: The data capture system digitizes the voltage at the output of the single-ended to differential converter (Fig. 2). The differential signal is fed to a 12-bit pipeline ADC, ADS6125. The sampling clock to the ADC is provided by a programmable clock generator IC, LMK61E2. The output frequency of the programmable clock generator can be varied from $10 \mathrm{MHz}$ to $1 \mathrm{GHz}$. For the target bandwidth of 1-10 MHz, this range of clock provides flexibility of sampling the signal from Nyquist rate to a high oversampled rate. The sampling frequency for any RUT is programmed during startup using the micro-controller, MSP430. Since the data transfer from the micro-controller is limited by transmission data rate, the 12 bit parallel digital data is stored in an onboard FIFO of depth $64 \mathrm{kB}$ and will be read in sequence.

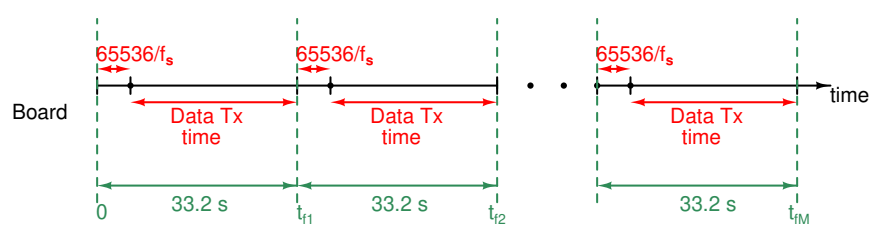

Fig. 3: Measurement protocol for data transfer.

The micro-controller waits till the server issues a ready command to initiate the data transfer. The micro-controller disables writing to the FIFO, once the FIFO is full. The microcontroller then initiates the data transfer to the back-end via UART or Bluetooth. The micro-controller ensures that further writing to the FIFO happens only once the entire data has been transferred. This is to ensure that we have chunks of $64 \mathrm{kB}$ continuous data even with different write and read clock frequencies. Fig. 3 shows the protocol followed for data transfer. Also, when the data is being written into the FIFO, the micro-controller remains idle. During this period, the micro-controller is used to measure the temperature with the help of a temperature sensor MCP9700. One hundred 


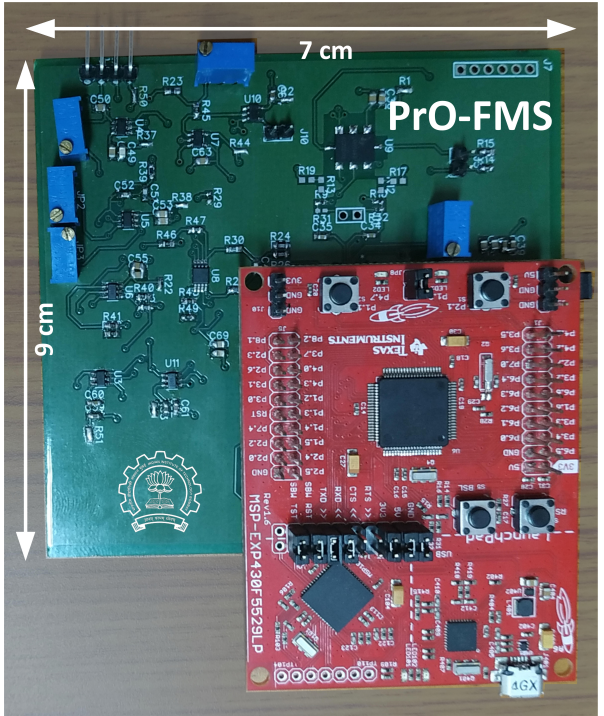

Fig. 4: The oscillator and data capture and transfer system of the proposed PrO-FMS (Dimensions: $7 \mathrm{~cm} \times 9 \mathrm{~cm}$ ).

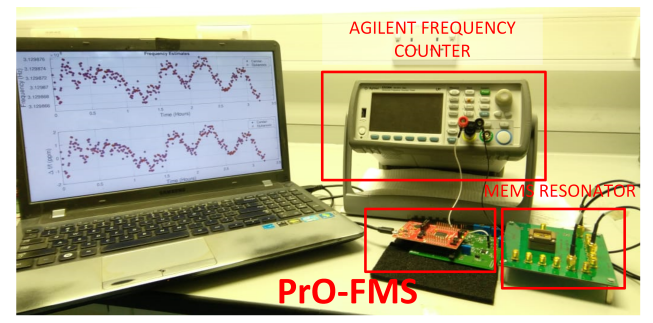

Fig. 5: PrO-FMS integrated with the MEMS RUT.

such readings are taken, while the FIFO is being written. To account for noise in the temperature readings, the microcontroller averages 100 temperature readings and transmits the temperature value along with the data from the FIFO. Therefore, for every data packet, we have one temperature value corresponding to one measurement interval. This ensures that the proposed system can be used to measure the frequency stability of the RUT with temperature and eventually correct the measured frequency with temperature.

It takes a total of 33.2 seconds for the complete process of data capture and successful transmission of the data. Consequently, new data sets are periodically collected after intervals of 33.2 seconds. To benchmark the board performance, the output of the comparator is buffered and fed to a frequency counter. The comparison results are shown in the experimental results section. Fig 4 shows the oscillator and the data capture system. Fig 5 shows the measurement setup integrated with a MEMS RUT.

3) Frequency Estimation: Very high accuracy frequency estimation algorithms [13]-[17] are run in a back-end (laptop for our experiments). These algorithms are classified into two groups: non-parametric methods, which estimate the signal frequency from the power spectral density and parametric methods, which use time domain-based approximations to estimate the signal frequency. These methods are illustrated in Fig. 6. [13] uses three bins from the signal DFT and calculates the frequency from these three bins. [14] again uses three samples from the signal DFT and estimates the frequency by using a parabolic interpolation. Clearly, the above algorithms require a highly computational platform to evaluate the $\mathrm{N}$ point DFT of the signal. [16] evaluates the time period with respect to the zero crossings and linear interpolation. This is less computationally intensive as it only requires to monitor the change in sign of the signal. [15], [17] discusses algorithms that use the time domain samples of the sinusoid to estimate its frequency. Both these techniques are used for single tone estimation. The paper compares the performance of all the five estimation techniques with the PrO-FMS with different signal to noise and distortion ratio (SNDR) and sampling frequency.

\section{CAlibration}

In applications where accuracy of the absolute frequency of measurement is important along with the change in the frequency, it is imperative to calibrate the system with a known frequency source for reference. For absolute frequency calibration, the oscillator circuit in the measurement system is operated in an open-loop with the TIA modified as a unity gain buffer, as shown in Fig. 7. A signal source, Agilent 33250-A with a frequency accuracy of $\pm 1 \mathrm{ppm}$ is used to calibrate the system with a sinusoid of known frequency. Simultaneously, the frequency is also measured with the tabletop Agilent53230A frequency counter using the on-board buffered test point. This frequency counter is a reciprocal counter with 12 digits accuracy and with noise rejection enabled. To measure the absolute frequency, the signal source is fixed at a particular frequency ( $\pm 1 \mathrm{ppm}$ accuracy) and measurements are made from the PrO-FMS for a long duration. The average frequency is then computed over this interval to reduce the effects of noise. This measurement protocol is shown in Fig. 8. The data is sampled and digitized by the ADC at the rate $\mathrm{f}_{s}$ and stored in the FIFO (depth $64 \mathrm{kB}$ ). The time required for writing $64 \mathrm{kB}$ data is $65536 / \mathrm{f}_{s}$, where $\mathrm{f}_{s}$ is the sampling frequency. The data in the FIFO is then transmitted to the backend server. This measurement is repeated over many such short data capture intervals. Simultaneously, the frequency is measured with the tabletop Agilent frequency counter. The gate time of the frequency counter is set to $65536 / f_{s}$ so as to match the data capture time of PrO-FMS. Hence, the frequency counter has one frequency value every $65536 / \mathrm{f}_{s}$. PrO-FMS has a measurement interval of $65536 / \mathrm{f}_{s}$ (same as frequency counter gate time) but a complete measurement and transfer time of 33.2 seconds for an individual frequency measurement. The additional frequency points from the frequency counter are decimated to have a one to one comparison with PrOFMS. The measurements are then repeated for different values of sampling frequency $\mathrm{f}_{s}$. It can be observed from the measurement results that the frequency counter readings are within the accuracy range of the signal-source while the PrOFMS has an offset in the absolute frequency for all the five algorithms as observed in Tables II, III and IV. This offset in the measured frequency is due to the offset in the sampling frequency of the PrO-FMS. The programmable clock has a minimal offset frequency which can now be measured with 


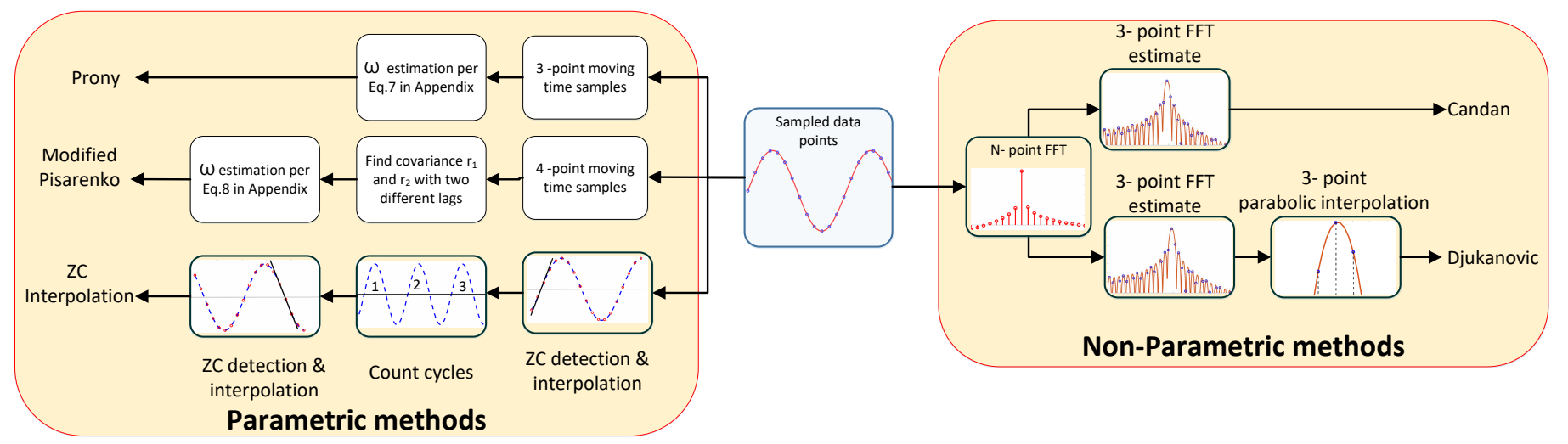

Fig. 6: Illustration of the frequency estimation methods.

the help of this calibration process. It can be observed from the tables II, III and IV that there is a fixed offset and a drift in the clock frequency. The drift in itself is a function of clock frequency and it is $5 \mathrm{~Hz}$ for every $10 \mathrm{MHz}$ increment in the clock frequency. This absolute frequency correction can be easily performed by providing the offset corrected clock frequency as the input to the frequency estimation algorithms. Tables II, III and IV show the results before and after offset

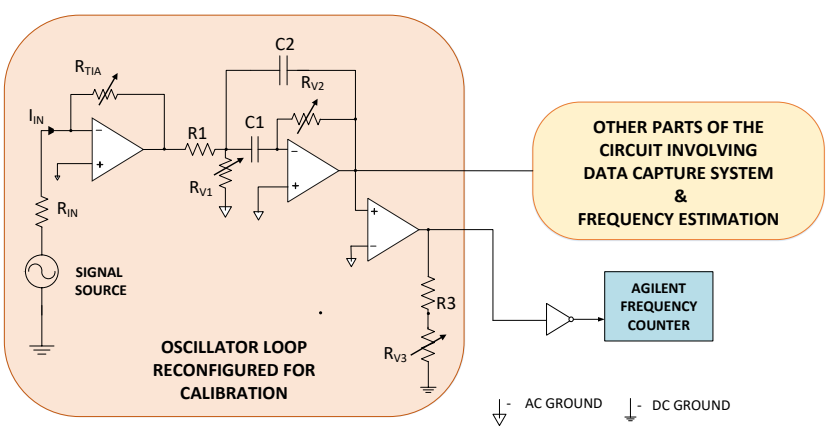

Fig. 7: Oscillator reconfigured in open loop for calibration.

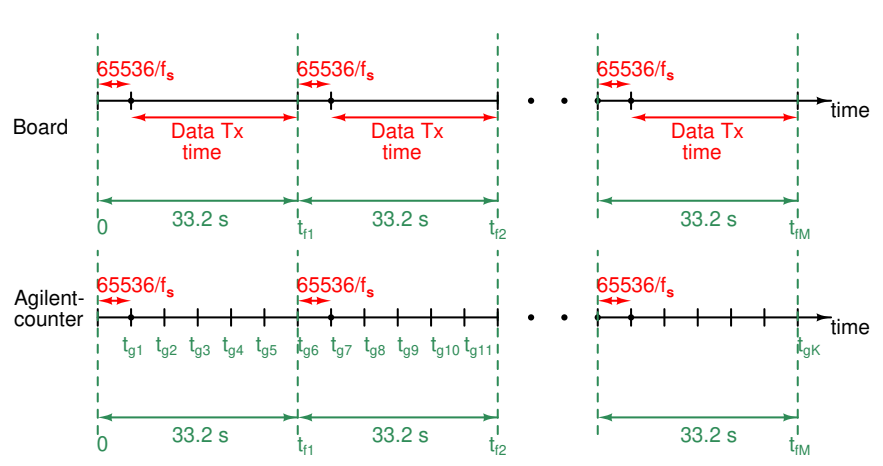

Fig. 8: Measurement protocol for long term measurements.

correction for three different sampling frequencies when the input frequency from the signal source is set at 3.1234567890 $\mathrm{MHz}$ ( \pm 1 ppm accuracy).

It can also be inferred that the two frequency estimate based algorithms and the zero crossing with linear interpolation have a higher accuracy of absolute frequency measurement as opposed to the remaining two time based algorithms.
TABLE II: Estimation with frequency correction for sampling frequency of $20 \mathrm{MHz}$.

\begin{tabular}{|c|c|c|}
\hline $\begin{array}{c}\text { Frequency Estimation } \\
\text { Methods } \\
\text { (double precision) }\end{array}$ & $\begin{array}{c}\mathbf{F}_{\text {estimate }} \\
\text { before correction (Hz) }\end{array}$ & $\begin{array}{c}\mathbf{F}_{\text {estimate }} \\
\text { after correction (Hz) }\end{array}$ \\
\cline { 2 - 3 } & $\mathbf{f}_{s}=\mathbf{2 0} \mathbf{~ M H z}$ & $\mathbf{f}_{s}=(\mathbf{2 0 M} \mathbf{- 1 5 3 . 7 5 ) ~ H z}$ \\
\hline Candan & 3123480.778593 & 3123456.766834 \\
\hline Djukanovic & 3123480.781182 & 3123456.769459 \\
\hline Prony & 3185399.541843 & 3185375.054084 \\
\hline M-Pisarenko & 3126962.752216 & 3126938.713690 \\
\hline ZC-Interpolation & 3123480.638858 & 3123456.627101 \\
\hline $\begin{array}{c}\text { Agilent-Counter } \\
\text { (accuracy upto 12 digits) }\end{array}$ & \multicolumn{2}{|c|}{3123457.937595} \\
\hline
\end{tabular}

TABLE III: Estimation with frequency correction for sampling frequency of $30 \mathrm{MHz}$.

\begin{tabular}{|c|c|c|}
\hline $\begin{array}{c}\text { Frequency Estimation } \\
\text { Methods } \\
\text { (double precision) }\end{array}$ & $\begin{array}{c}\mathbf{F}_{\text {estimate }} \\
\text { before correction (Hz) }\end{array}$ & $\begin{array}{c}\mathbf{F}_{\text {estimate }} \\
\text { after correction (Hz) }\end{array}$ \\
\cline { 2 - 3 } & $\mathbf{f}_{s}=\mathbf{3 0} \mathbf{~ M H z}$ & $\left.\mathbf{f}_{s}=\mathbf{( 3 0 M}-\mathbf{1 5 8 . 7 5}\right) \mathbf{H z}$ \\
\hline Candan & 3123481.283441 & 3123456.490809 \\
\hline Djukanovic & 3123481.287053 & 3123456.494421 \\
\hline Prony & 3221589.585249 & 3221564.013881 \\
\hline M-Pisarenko & 3145771.122663 & 3145746.153104 \\
\hline ZC-Interpolation & 3123481.269600 & 3123456.476968 \\
\hline $\begin{array}{c}\text { Agilent-Counter } \\
\text { (accuracy upto 12 digits) }\end{array}$ & \multicolumn{2}{|c|}{3123458.005980} \\
\hline
\end{tabular}

\section{EXPERIMENTAL RESULTS}

The PrO-FMS was used to measure the absolute frequency and the deviation for two different quartz crystals and a MEMS RUT in two different modes [19]. A sample set of data points captured from the system is shown in Fig. 10. The measurements are divided into two sections, namely short term and long term measurements. The aim of the short term measurement experiment, is to measure the RMSE of the frequency measurements over short time intervals with

TABLE IV: Estimation with frequency correction for sampling frequency of $40 \mathrm{MHz}$.

\begin{tabular}{|c|c|c|}
\hline $\begin{array}{c}\text { Frequency Estimation } \\
\text { Methods }\end{array}$ & $\begin{array}{l}\mathbf{F}_{\text {estimate }} \\
\text { before correction }(\mathbf{H z})\end{array}$ & $\begin{array}{l}\quad \mathbf{F}_{\text {estimate }} \\
\text { after correction }(\mathbf{H z})\end{array}$ \\
\hline & $\mathrm{f}_{s}=40 \mathrm{MHz}$ & $\mathrm{f}_{s}=(40 \mathrm{M}-163.75) \mathrm{Hz}$ \\
\hline Candan & 3123482.225056 & 3123456.651546 \\
\hline Djukanovic & 3123482.200386 & 3123456.626877 \\
\hline Prony & 3235317.556215 & 3235291.067052 \\
\hline M-Pisarenko & 3172313.566987 & 3172287.593669 \\
\hline ZC-Interpolation & 3123480.944348 & 3123455.370848 \\
\hline $\begin{array}{c}\text { Agilent-Counter } \\
\text { (accuracy upto } 12 \text { digits) }\end{array}$ & \multicolumn{2}{|c|}{3123457.832081} \\
\hline
\end{tabular}




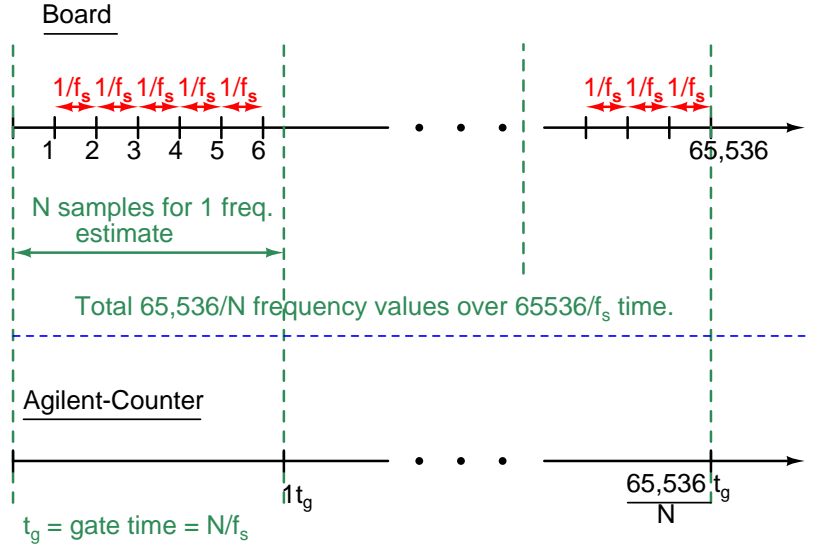

Fig. 9: Measurement protocol for short term measurements.

different gate times and different SNDR. This provides an insight as to how the different frequency estimation algorithms perform with varying gate times and SNR when compared to a tabletop frequency counter with the same gate time. Short term measurements will be devoid of any long term frequency drifts that could arise due to variety of reasons like temperature and humidity. The long term measurements on the other hand are performed to observe the long term frequency drift with temperature and any other environment change. Frequency corrections with temperature could then be employed in the long term measurements with the temperature data.

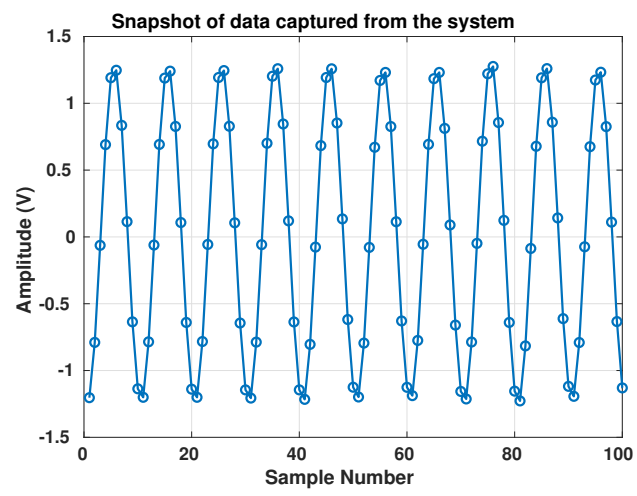

Fig. 10: Snapshot of data captured from the system with 40 $\mathrm{MHz}$ sampling frequency.

\section{A. Short term measurements}

After the baseline calibration, PrO-FMS can be used to characterize resonators in the bandwidth of 1-10 MHz. To compare, the data is acquired simultaneously from the PrOFMS and the Agilent frequency counter 33250A. The protocol for short term measurements is shown in Fig. 9.

In the data capture system, a sample is collected every $1 / \mathrm{f}_{s}$ second, where $\mathrm{f}_{s}$ is the sampling frequency. The samples are continuously stored in a FIFO of depth $64 \mathrm{kB}$. "N" such samples are used to estimate one frequency value. To make a fair comparison with PrO-FMS, this would correspond to a gate time of $\mathrm{N} / \mathrm{f}_{s}$ seconds in the frequency counter. The measurements are repeated for the various values of
"N". The gate time is changed accordingly in the frequency counter for every measurement. The frequency of the signal is estimated from the data captured using five different frequency estimation techniques, [13]-[17]. The RMSE is then estimated as the standard deviation of $\Delta f / f$ for the frequency counter and the five estimation methods. The measurement results for two different RUTs with different resonant frequencies and a different signal to noise ratio (at the input of the ADC) are discussed.

1) $4 \mathrm{MHz}$ quartz crystal: A quartz crystal with a resonant frequency of $4 \mathrm{MHz}$ is characterized with the PrO-FMS. The measurements are repeated for two different SNDR values (measured at the output of the single-ended to differential converter). The Fig. 11 shows the Allan deviation plot [20], [21] for the $4 \mathrm{MHz}$ crystal measured with a tabletop frequency counter. The crystal has a stability of $2 \mathrm{ppb}$ as seen from the Allan deviation plot. The Fig. 13 shows the RMSE of each frequency estimation technique vs. gate time. It is evident from the plots that the RMSE for the two time-based methods namely Prony and Modified Pisarenko does not do well compared to the Agilent counter. The Candan method has the lowest RMSE. However, with a higher gate time, the performance of the Djukanovic method approaches that of Candan. The zero crossing interpolation does better at higher sampling frequencies due to the reduction in interpolation error. From all the plots, it is noticeable that higher SNR leads to lower RMSE as expected. It is also observed that the frequency estimations provided by Candan method has lower RMSE than the Agilent counter for the same gate time even with a low sampling rate of $20 \mathrm{MHz}$.

2) $8 \mathbf{M H z}$ quartz crystal: Subsequently, measurements are done for a quartz crystal with a resonant frequency of $8 \mathrm{MHz}$ is with the Pro-FMS. The measurements are repeated again for two different SNDR values for three different sampling rates. As seen from the Fig. 11, the Allan deviation plot for the 8 $\mathrm{MHz}$ crystal shows a stability of $3.3 \mathrm{ppb}$. Fig. 14 shows the RMSE of each frequency estimation technique vs. gate time. The inferences are similar to that of $4 \mathrm{MHz}$ crystal except for the interpolation method. Due to the reduction in oversampling ratio (OSR), the interpolation error increases for an $8 \mathrm{MHz}$ crystal. Also, it can be observed that SNDR does not have any effect since interpolation error dominates at higher gate times.

3) MEMS resonator: The PrO-FMS is used to measure the resonant modes of a MEMS RUT. The MEMS has two different modes of resonance: the square extensional (SE) mode and the butterfly (BF) mode [19]. The open-loop characterization of the MEMS is done using a network analyzer and the transmission magnitude plot is shown in Fig. 12. The openloop characteristics of the MEMS RUT indicates clearly that it has a square extensional mode at $3.02 \mathrm{MHz}(\mathrm{Q}=2100)$ and a butterfly mode at $3.13 \mathrm{MHz}(\mathrm{Q}=3800)$. The Allan deviation plot for the two modes of MEMS are shown in Fig.11. It has a stability of $44 \mathrm{ppb}$ and $18 \mathrm{ppb}$ in the SE and the BF mode, respectively. The figures 15 and 16 shows the RMSE of frequency vs. gate time when excited in the square extensional and butterfly mode, respectively. It is evident from the plots that the RMSE for the two time-based methods namely Prony 


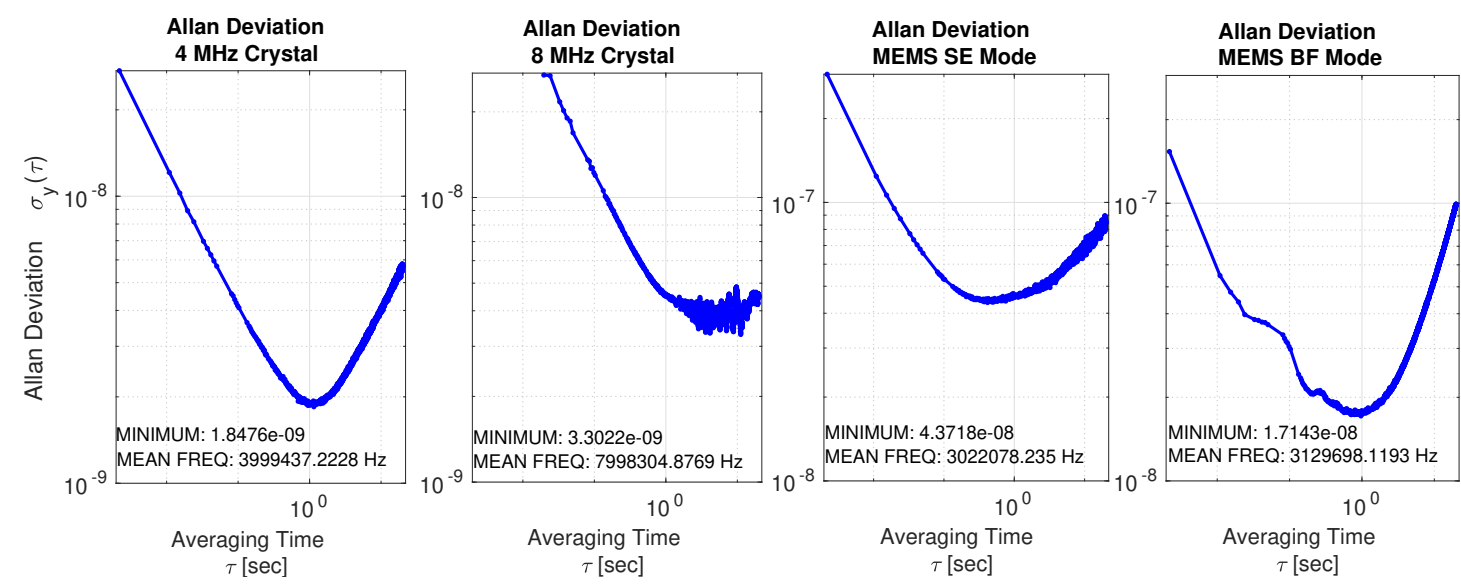

Fig. 11: Allan deviation plots: (a) $4 \mathrm{MHz}$ Quartz crystal (b) $8 \mathrm{MHz}$ Quartz crystal (c) MEMS SE mode (d) MEMS BF mode.

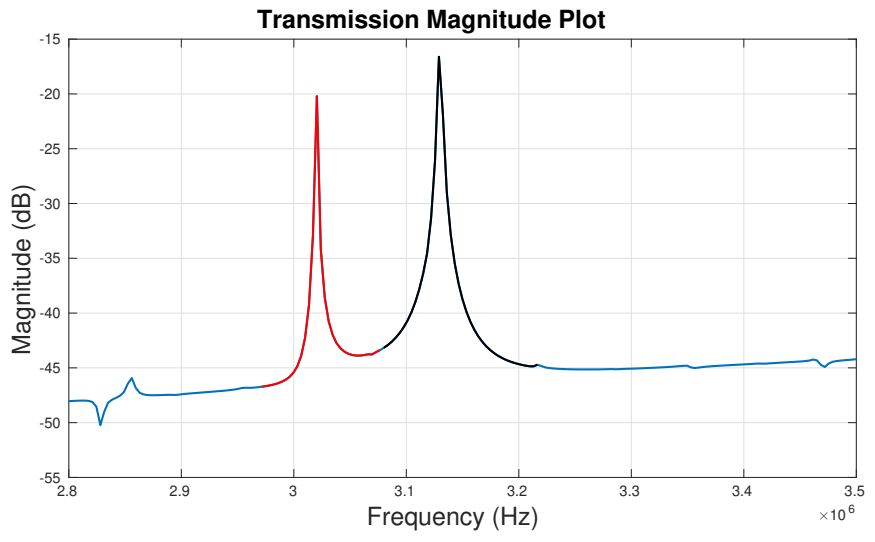

Fig. 12: Open loop characteristics of MEMS RUT.

and Modified Pisarenko does not do well compared to the Agilent counter. The Candan method has the lowest RMSE but as the gate time increases, the Djukanovic method performance approaches that of Candan. The zero-crossing interpolation does better at higher sampling frequencies since this reduces the error in interpolation. It is also observed that the frequency estimations from PrO-FMS provide lower RMSE for Candan method than the Agilent counter for the same gate time.

\section{B. Long term measurements}

The protocol for long term measurements is the same as the protocol followed during the absolute frequency calibration. The data capture system collects samples every $1 / \mathrm{f}_{s}$ seconds. The data capture board collects a total of 65536 samples.

All of the 65536 samples are used to estimate one frequency value. This corresponds to a gate time of $65536 / \mathrm{f}_{s}$ for the frequency counter. The measurement protocol is the same as shown in Fig. 8. This measurement is conducted for a long duration with a sampling frequency of $20 \mathrm{MHz}$ and a signal to noise ratio of $40 \mathrm{~dB}$. The frequency estimates are then obtained from the back-end server along with the error which is evaluated as per Eq.2, where $f_{k}$ is $k^{\text {th }}$ instantaneous frequency estimate and $f_{\text {mean }}$ is the mean of all the $f_{k}$ values.

$$
\frac{\Delta f_{k}}{f}=\frac{f_{\text {mean }}-f_{k}}{f_{\text {mean }}}
$$

The RMSE is evaluated by taking the standard deviation of $\Delta f_{k} / f$.

1) $4 \mathrm{MHz}$ quartz crystal: From the short term measurements, it is observed that the DFT based frequency estimation algorithms provide better resolution compared to the other algorithms. Consequently, a quartz crystal with a resonant frequency of $4 \mathrm{MHz}$ is characterized for a longer time duration $(\approx 30$ minutes $)$ using the Pro-FMS with the DFT based frequency estimation techniques. The long term resonant frequency behavior of quartz crystals is shown in Fig. 17. Table $\mathrm{V}$ shows the RMSE of the estimated frequency for the $4 \mathrm{MHz}$ quartz crystal. It is clearly evident from the table that the Pro-FMS is able to achieve resolution of $0.1 \mathrm{ppm}$ RMSE.

TABLE V: RMSE of frequency measurement for $4 \mathrm{MHz}$ and $8 \mathrm{MHz}$ crystals (long-term measurements).

\begin{tabular}{|c|c|c|}
\hline $\begin{array}{c}\text { Frequency estimation method } \\
\text { (double precision) }\end{array}$ & $\begin{array}{c}\text { 4 MHz crystal } \\
\text { RMSE (ppm) }\end{array}$ & $\begin{array}{c}\text { 8 MHz crystal } \\
\text { RMSE (ppm) }\end{array}$ \\
\hline Candan & 0.098406 & 0.048527 \\
\hline Djukanovic & 0.098208 & 0.048200 \\
\hline \multicolumn{2}{|c|}{$\begin{array}{c}\text { Frequency estimation using Agilent frequency } \\
\text { counter (accuracy upto 12 digits) }\end{array}$} \\
\hline Agilent counter & 0.108134 & 0.081420 \\
\hline
\end{tabular}

2) $8 \mathrm{MHz}$ quartz crystals: A quartz crystal with a resonant frequency of $8 \mathrm{MHz}$ is characterized for a longer time duration $(\approx 30$ minutes) using the Pro-FMS with the DFT based frequency estimation techniques. The long term frequency behavior of quartz crystal is shown in Fig. 18. Table V shows the RMSE of the frequency estimated for the $8 \mathrm{MHz}$ quartz crystal. It is clearly evident from Table V that the Pro-FMS is able to achieve a resolution of $0.1 \mathrm{ppm}$ RMSE.

3) MEMS resonator: The frequency of the MEMS RUT with two different modes of resonance, the SE and the BF mode [19], is monitored using PrO-FMS. Figures 19 and 20 show the long term variation of frequency (with the DFT based frequency estimation techniques) over time when excited in the SE and BF mode, respectively in a controlled lab environment. Tables VI and VI show the RMSE of the estimated frequency for the MEMS RUT when excited in $\mathrm{SE}$ and $\mathrm{BF}$ mode, respectively. Clearly, from the long term 

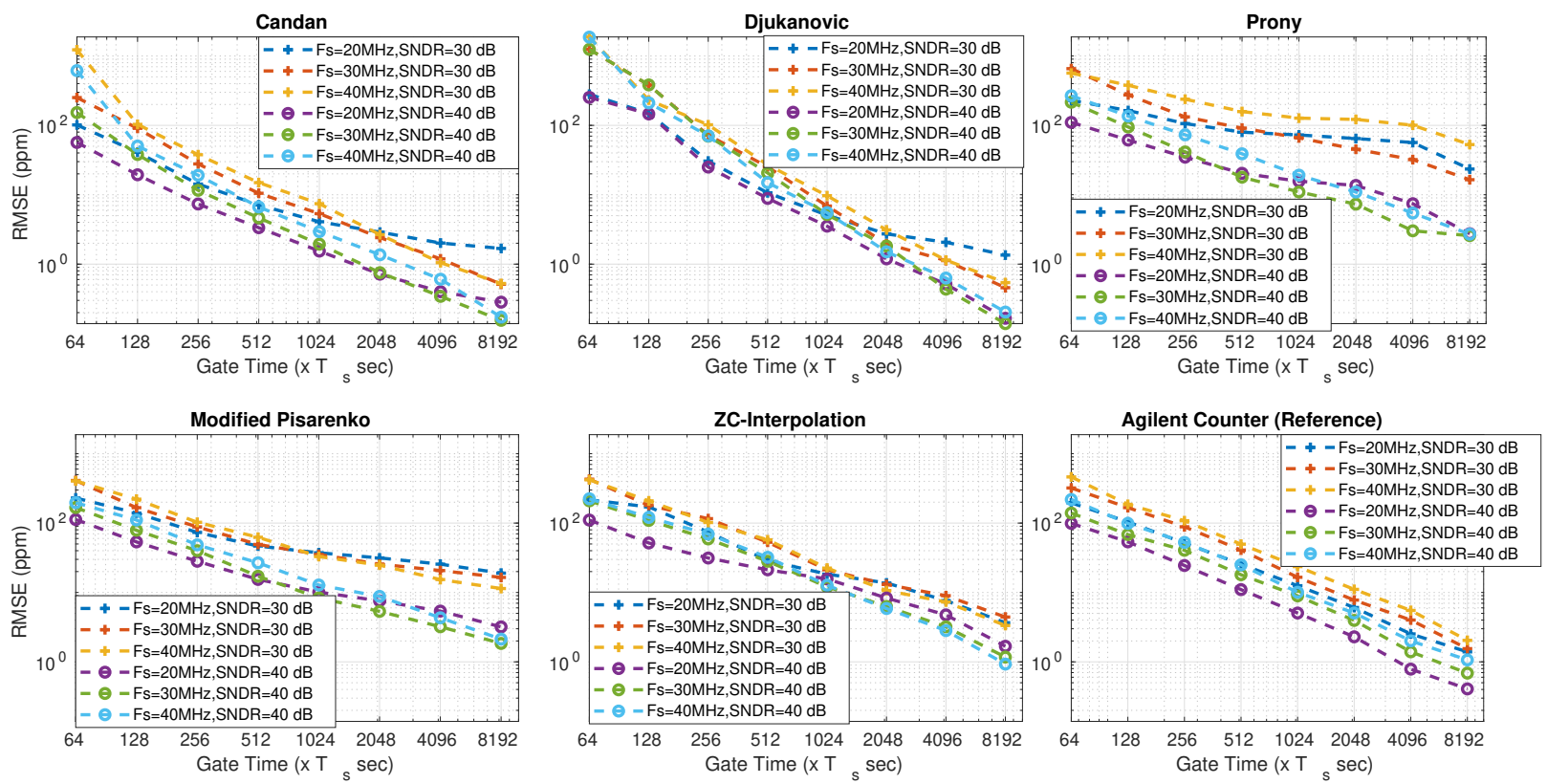

Fig. 13: RMSE of frequency vs Gate time for $4 \mathrm{MHz}$ quartz crystal (short-term measurements).
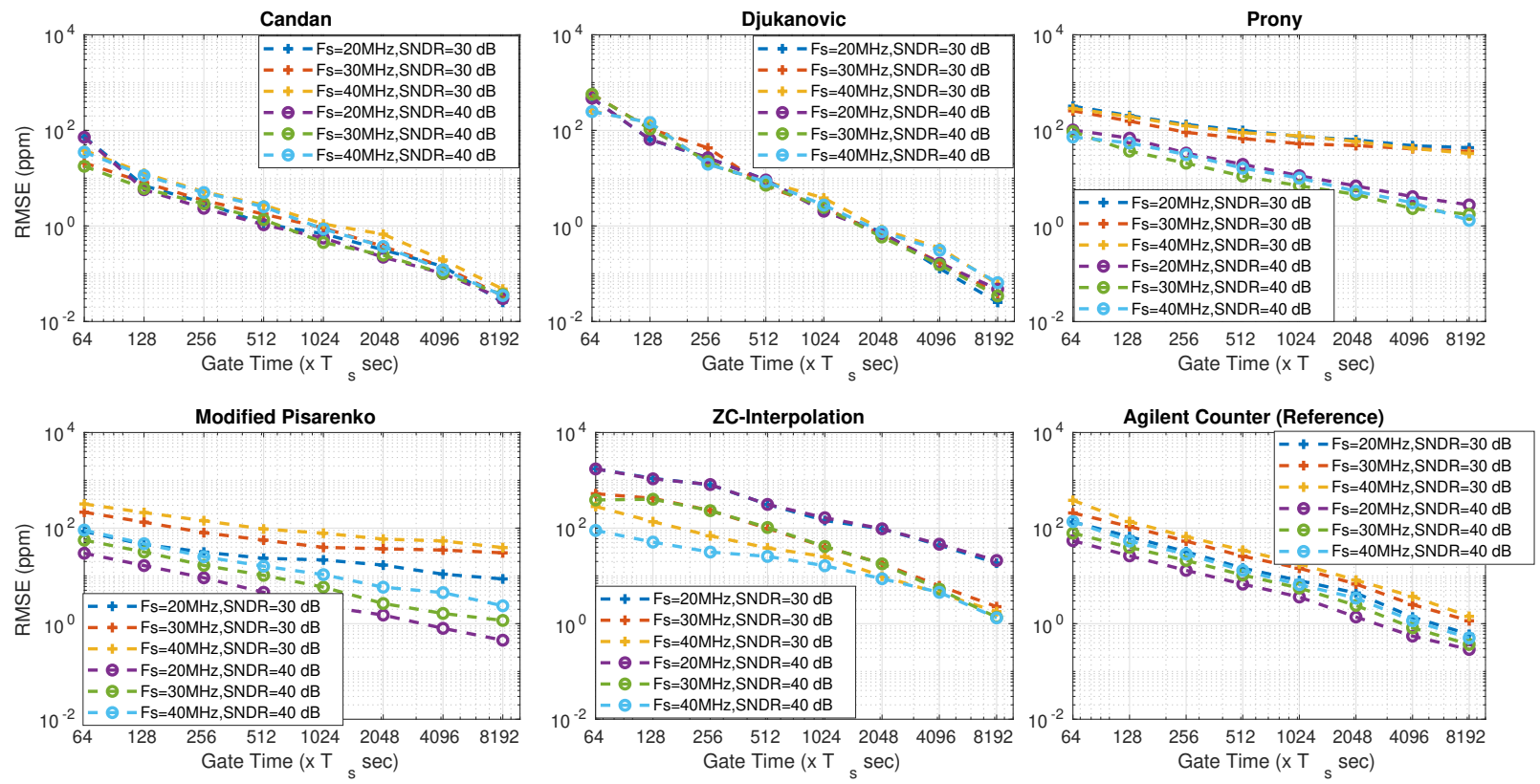

Fig. 14: RMSE of frequency vs Gate time for $8 \mathrm{MHz}$ quartz crystal (short-term measurements).

measurements, the DFT based frequency estimation algorithms show lower variance about the mean frequency compared to the Agilent counter for the same gate time.

TABLE VI: RMSE of frequency measurement for MEMS RUT in SE and BF mode of excitation (long-term measurements).

\begin{tabular}{|c|c|c|}
\hline $\begin{array}{c}\text { Frequency estimation method } \\
\text { (double precision) }\end{array}$ & $\begin{array}{c}\text { SE mode } \\
\text { RMSE (ppm) }\end{array}$ & $\begin{array}{c}\text { BF mode } \\
\text { RMSE (ppm) }\end{array}$ \\
\hline Candan & 0.544281 & 0.248061 \\
\hline Djukanovic & 0.544521 & 0.246903 \\
\hline $\begin{array}{c}\text { Frequency estimation using Agilent frequency } \\
\text { counter (accuracy upto 12 digits) }\end{array}$ \\
\hline Agilent counter & 0.539488 & 0.271626 \\
\hline
\end{tabular}

Quartz crystals show higher frequency stability with respect to the temperature as compared to MEMS resonators. The proposed system has an additional feature of logging in the ambient temperature while transferring the data. This temperature information can be used to correct the frequency drift in MEMS resonator due to temperature variations. Thus long measurements $(\approx 3.25 \mathrm{hrs})$ are conducted to characterize the MEMS resonator for temperature variations. Figures 21 and 22 shows the frequency drift with time and the captured temperature data for $\mathrm{SE}$ and $\mathrm{BF}$ mode, respectively. Consequently, for simplicity a third-order polynomial of the form, given by Eq. 3 is chosen to correct for the temperature dependence of the MEMS RUT. Table VII shows the values of the coefficients 

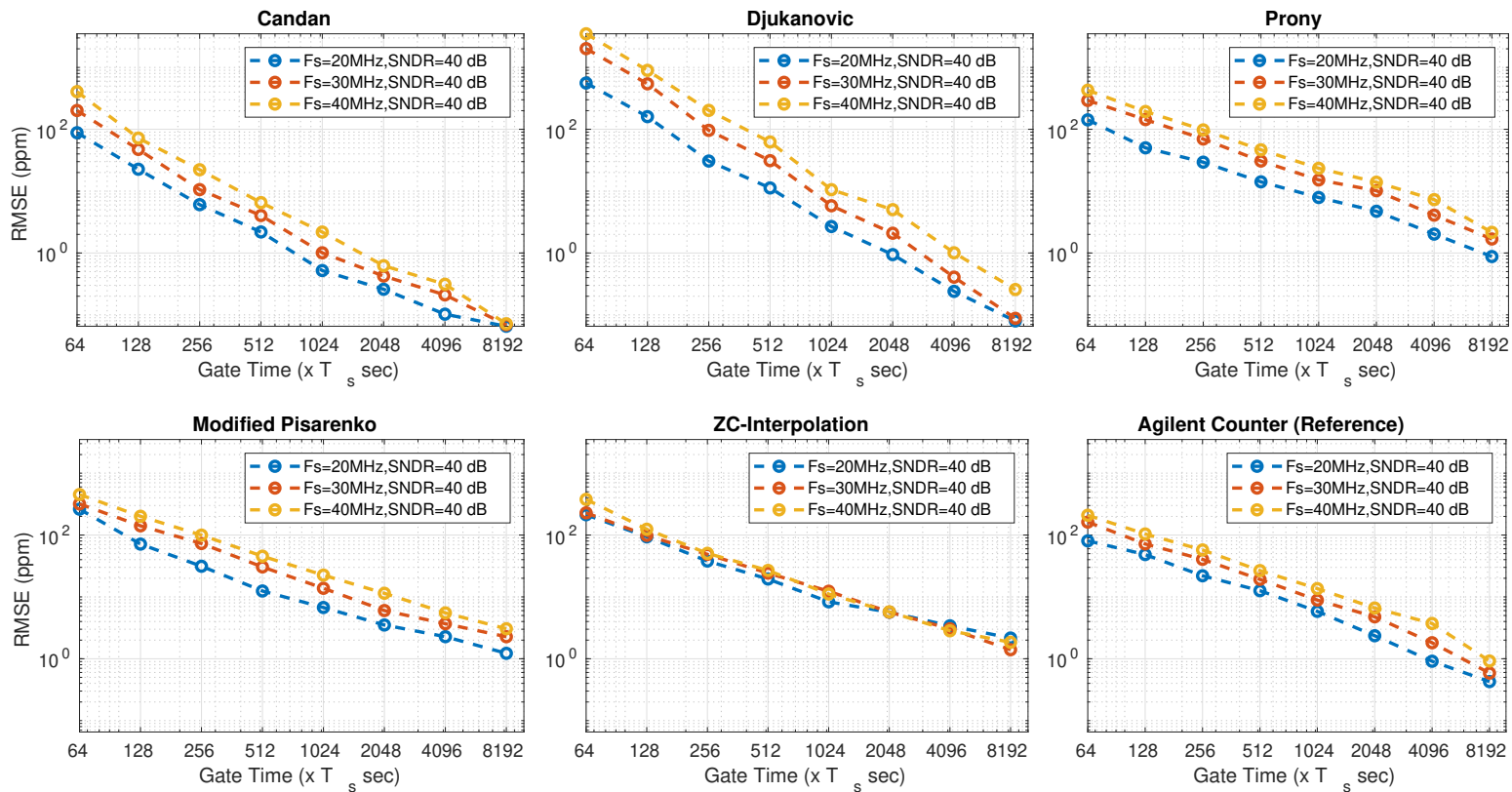

Fig. 15: RMSE of frequency vs Gate time for MEMS square extensional mode (short-term measurements).
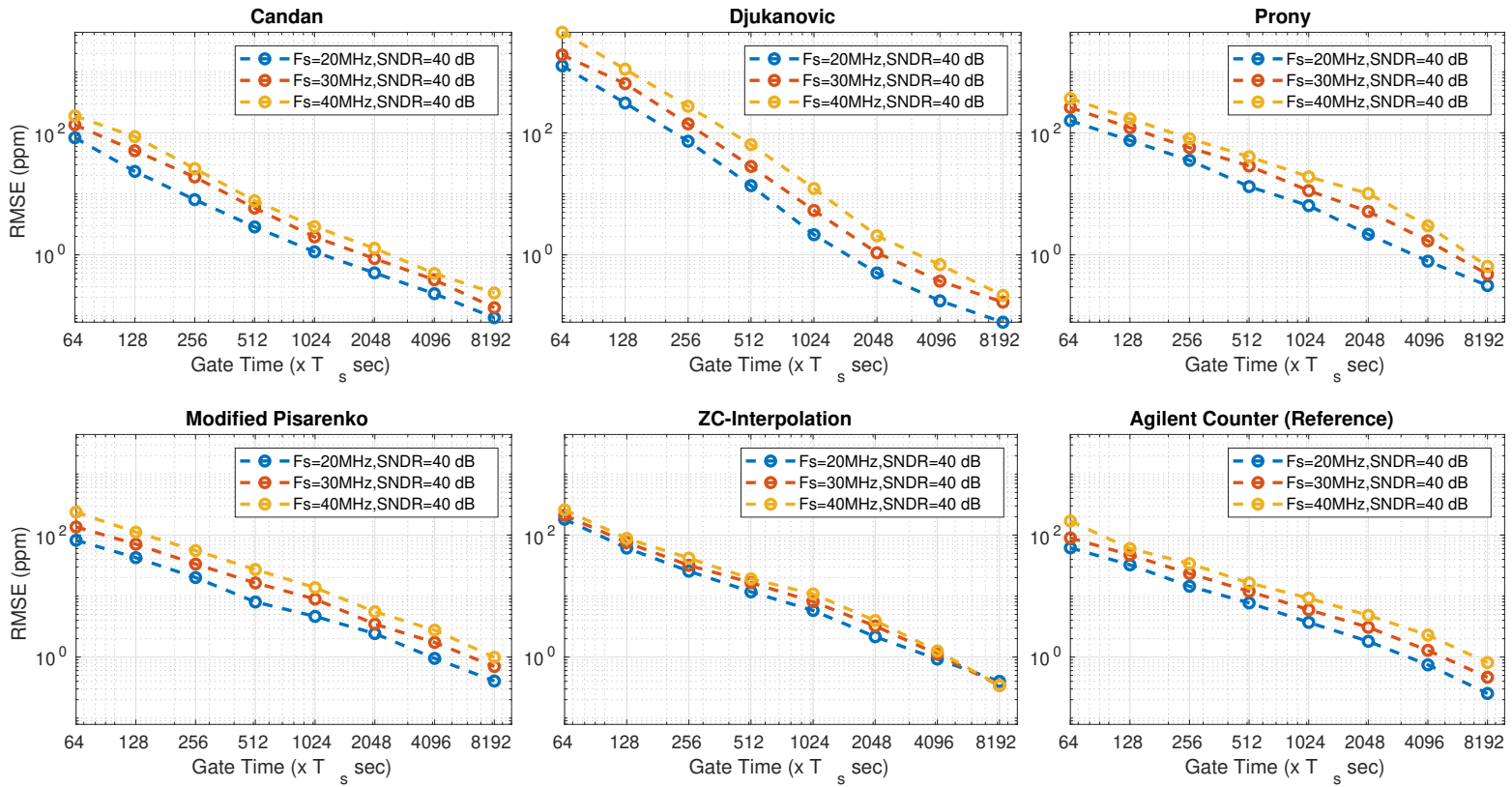

Fig. 16: RMSE of frequency vs Gate time for MEMS butterfly mode (short-term measurements).

for the SE and BF mode of the MEMS RUT. Figures 23 and 24 show the estimated frequency after a third order polynomial temperature correction. It is evident that the maximum value of $\Delta f / f$ is within $\pm 2 \mathrm{ppm}$.

$$
\begin{gathered}
f_{i, \text { new }}=f_{i}-\left[k_{t e m p 1} \Delta T_{i}+k_{t e m p} \Delta T_{i}^{2}+k_{t e m p} \Delta T_{i}^{3}\right] \\
\Delta T_{i}=T_{\text {avg }}-T_{i}
\end{gathered}
$$

\section{CONCLusions AND Future Work}

\section{A. Conclusion}

The implementation of PrO-FMS, a portable 0.1 ppm RMSE resolution programmable frequency measurement system for
TABLE VII: Polynomial coefficients for temperature compensation for MEMS RUT in SE and butterfly mode.

\begin{tabular}{|c|c|c|}
\hline \multirow{2}{*}{ Coefficient } & \multicolumn{2}{|c|}{ Coefficient value } \\
\cline { 2 - 3 } & SE mode & Butterfly mode \\
\hline$k_{t e m p 1}$ & $\mathbf{1 2 5 ~} \mathrm{Hz} /{ }^{\circ} \mathrm{C}$ & $\mathbf{1 1 0 ~} \mathrm{Hz} /{ }^{\circ} \mathrm{C}$ \\
\hline$k_{t e m p 2}$ & $\mathbf{- 4 5 ~} \mathrm{Hz} /{ }^{\circ} \mathrm{C}$ & $\mathbf{- 4 5 ~} \mathrm{Hz} /{ }^{\circ} \mathrm{C}$ \\
\hline$k_{t e m p} 3$ & $\mathbf{- 1 5 0 ~} \mathrm{Hz} /{ }^{\circ} \mathrm{C}$ & $\mathbf{- 1 5 0 ~} \mathrm{Hz} /{ }^{\circ} \mathrm{C}$ \\
\hline
\end{tabular}

high resolution, low measurement interval resonator applications is presented. The proposed system has a tunable oscillator loop which can be reconfigured based on the resonator under test to support a range of resonant devices (1-10 MHz bandwidth). The system has a programmable 

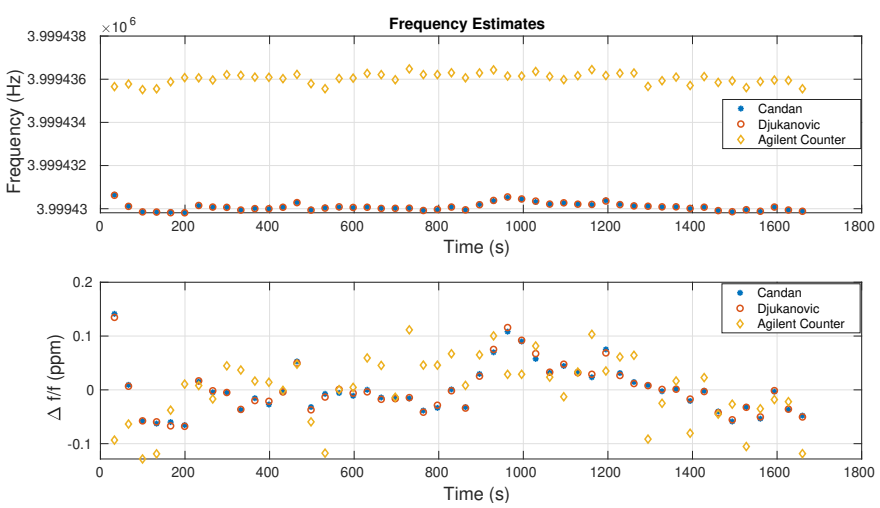

Fig. 17: Long-term resonant frequency behaviour of $4 \mathrm{MHz}$ quartz crystal over time (30 minutes).
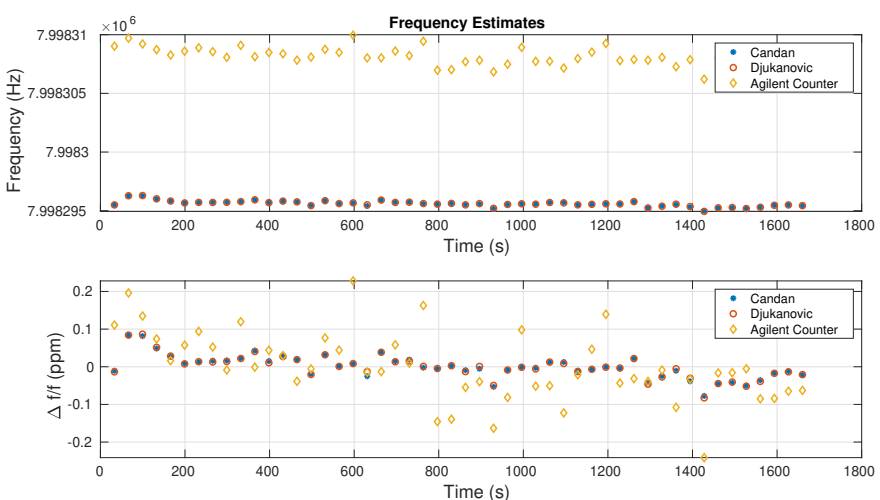

Fig. 18: Long-term resonant frequency behaviour of $8 \mathrm{MHz}$ quartz crystal over time (30 minutes).
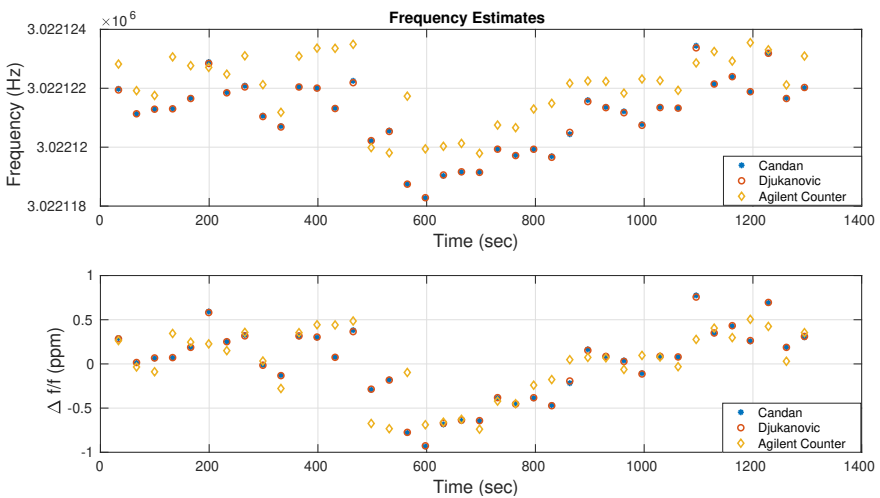

Fig. 19: Long-term resonant frequency behaviour of MEMS RUT in square extensional mode over time (30 minutes).

data capture module and a data transmission module in line with sensor node applications. A back-end server/computation engine estimates the frequency values. Five different frequency estimation algorithms were demonstrated. Baseline absolute frequency calibration and measurement results were discussed and demonstrated for two crystals and two modes of a MEMS resonator. The proposed system was compared with a tabletop Agilent-53230A reciprocal frequency counter with an accuracy of 12 digits and noise rejection enabled. Short-term and long term measurement results were presented for different resonant devices. The measurement results show that the performance
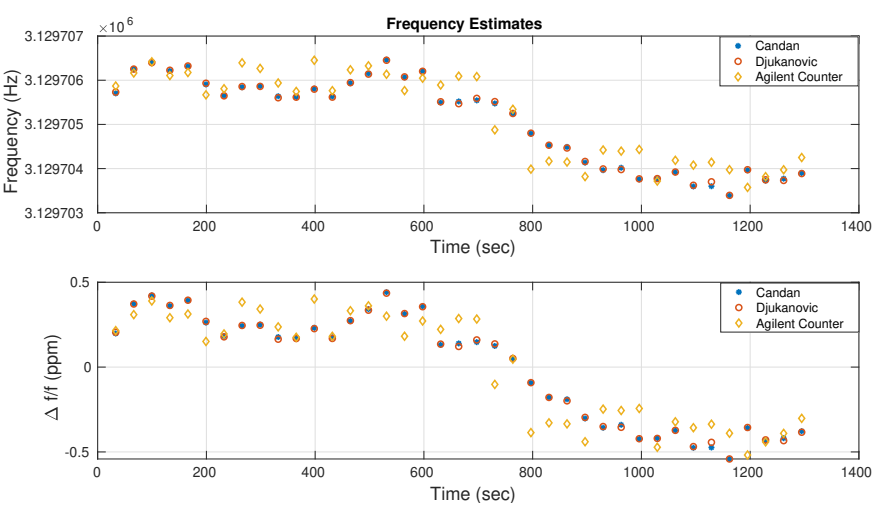

Fig. 20: Long-term resonant frequency behaviour of MEMS RUT in butterfly mode over time (30 minutes).
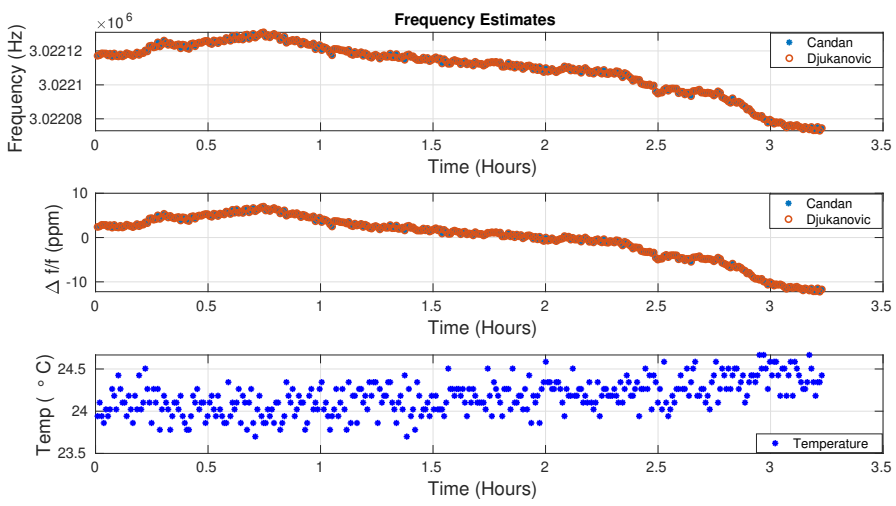

Fig. 21: Resonant frequency variation of the MEMS RUT with time and temperature in square extensional mode.
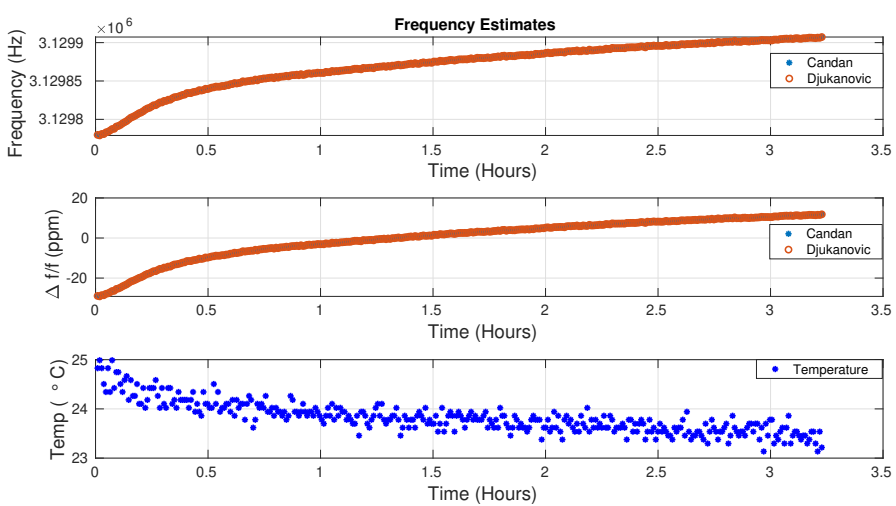

Fig. 22: Resonant frequency of the MEMS RUT with time and temperature in butterfly mode.

of the PrO-FMS is better than the Agilent counter for the same gate time. Measurement results also show that the PrOFMS can measure frequency upto $0.1 \mathrm{ppm}$ RMSE. The system also provides an efficient platform to adapt different frequency estimation techniques. Thus for a particular application, the number of data points to be captured and the type of algorithm can be decided solely on the accuracy requirements. Therefore the board, along with the back end computation, can adapt according to the needs of the application. 

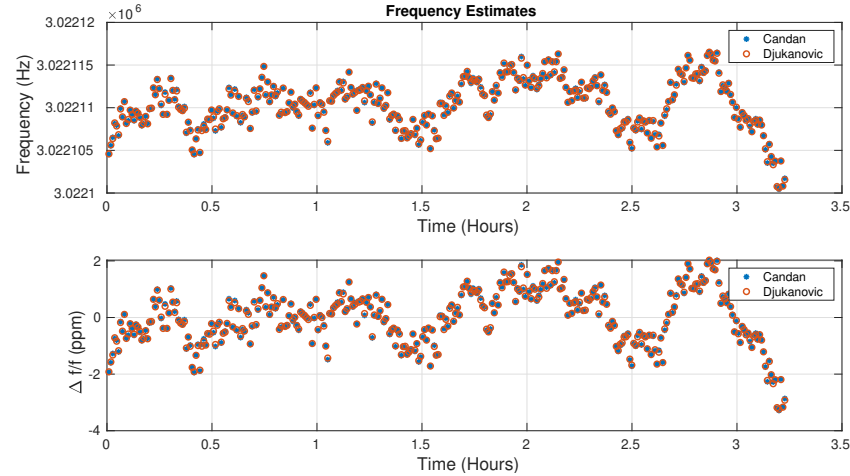

Fig. 23: Resonant frequency behaviour of MEMS RUT in square extensional mode after temperature correction.
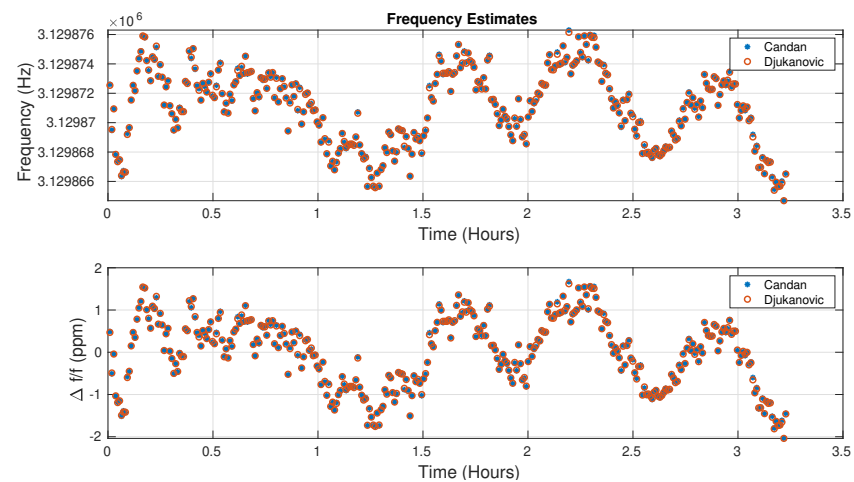

Fig. 24: Resonant frequency of MEMS RUT in butterfly mode after temperature correction.

\section{B. Future Work}

The bottleneck in faster frequency measurements in the PrO-FMS stems from the low data transmission rate. Faster data transmission techniques need to be utilized to increase the throughput of the measurements to cater a wide range of applications. The system can be scaled to operate for RUTs with bandwidth more than $10 \mathrm{MHz}$. The data capture board is programmable to support this wider range.

\section{ACKNOWLEDGMENT}

The authors would like to acknowledge the financial support from the Department of Science and Technology, Government of India and British Council, UK, under the framework of a DST-UKIERI joint research collaboration.

\section{REFERENCES}

[1] C. T. . Nguyen, "MEMS technology for timing and frequency control," IEEE Trans. on Ultrasonics, Ferroelectrics, and Frequency Control, vol. 54, no. 2, pp. 251-270, February 2007.

[2] F. Ayazi, "Multi-DOF inertial MEMS: From gaming to dead reckoning," in 2011 16th International Solid-State Sensors, Actuators and Microsystems Conf., June 2011, pp. 2805-2808.

[3] C. Li, H. Wen, S. Wisher, A. Norouzpour-Shirazi, J. Lei, H. Chen, and F. Ayazi, "An FPGA-Based Interface System for High Frequency Bulk-Acoustic-Wave (BAW) Micro-Gyroscopes with In-Run Automatic Mode-Matching," IEEE Trans. on Instr. and Measurement, 2019.

[4] A. T. Zielinski, M. Kalberer, R. L. Jones, A. Prasad, and A. A. Seshia, "Particulate mass sensing with piezoelectric bulk acoustic mode resonators," in 2016 IEEE International Frequency Control Symposium (IFCS), May 2016, pp. 1-6.
[5] A. Prasad, A. A. Seshia, A. T. Zielinski, M. Kalberer, and R. L. Jones, "Studying particulate adsorption by drying droplets on a microfabricated electro-acoustic resonator," in 2014 European Frequency and Time Forum (EFTF), June 2014, pp. 28-31.

[6] Agilent 53230A. RF/Universal Frequency Counter/Timers. [Online]. Available: https://literature.cdn.keysight.com/litweb/pdf/59906283EN.pdf?id=1942617

[7] K. R.Szplet, Z.Jachna and J.Kalisz, "High Precision Time and Frequency Counter for Mobile Applications," WSEAS Trans. on Circuits and Systems, vol. 9, no. 6, June 2010.

[8] L. Iafolla et al., "FPGA-based time to digital converter and data acquisition system for high energy tagger of KLOE-2 experiment," Nucl. Instrum. Methods Phys. Res. A, Accel. Spectrom. Detect. Assoc. Equip, vol. 718, no. 7, pp. 213-214, 2012.

[9] J. Kalisz, R. Szplet, R. Pelka, and A. Poniecki, "Single-chip interpolating time counter with 200-ps resolution and 43-s range," IEEE Trans. on Instr. and Measurement, vol. 46, no. 4, pp. 851-856, Aug 1997.

[10] R. Szplet, J. Kalisz, and R. Szymanowski, "Interpolating time counter with 100 ps resolution on a single FPGA device," IEEE Trans. on Instr. and Measurement, vol. 49, no. 4, pp. 879-883, Aug 2000.

[11] Jian Song, Qi An, and Shubin Liu, "A high-resolution time-to-digital converter implemented in field-programmable-gate-arrays," IEEE Trans. on Nuclear Science, vol. 53, no. 1, pp. 236-241, Feb 2006

[12] Q. Shen, S. Liu, B. Qi, Q. An, S. Liao, P. Shang, C. Peng, and W. Liu, "A 1.7 ps Equivalent Bin Size and 4.2 ps RMS FPGA TDC Based on Multichain Measurements Averaging Method," IEEE Trans. on Nuclear Science, vol. 62, no. 3, pp. 947-954, June 2015.

[13] C. Candan, "Analysis and Further Improvement of Fine Resolution Frequency Estimation Method From Three DFT Samples," IEEE Signal Processing Letters, vol. 20, no. 9, pp. 913-916, Sep. 2013.

[14] S. Djukanović, T. Popović, and A. Mitrović, "Precise sinusoid frequency estimation based on parabolic interpolation," in 2016 24th Telecommunications Forum (TELFOR), Nov 2016, pp. 1-4.

[15] D. W. Tufts and P. D. Fiore, "Simple, effective estimation of frequency based on Prony's method," in 1996 IEEE International Conf. on Acoustics, Speech, and Signal Processing Conf. Proceedings, vol. 5, May 1996, pp. 2801-2804 vol. 5.

[16] V. Friedman, "A zero crossing algorithm for the estimation of the frequency of a single sinusoid in white noise," IEEE Trans. on Signal Processing, vol. 42, no. 6, pp. 1565-1569, June 1994.

[17] M. D. Kusljevic, "A simple recursive algorithm for frequency estimation," IEEE Trans. on Instrumentation and Measurement, vol. 53, no. 2, pp. 335-340, April 2004

[18] J. E. . Lee, B. Bahreyni, Y. Zhu, and A. A. Seshia, "A Single-CrystalSilicon Bulk-Acoustic-Mode Microresonator Oscillator," IEEE Electron Device Letters, vol. 29, no. 7, pp. 701-703, July 2008.

[19] A. Prasad, J. Charmet, and A. A. Seshia, "Simultaneous interrogation of high-q modes in a piezoelectric-on-silicon micromechanical resonator," Sensors and Actuators A: Physical, vol. 238, pp. $207-214,2016 . \quad$ [Online]. Available: http://www.sciencedirect.com/science/article/pii/S0924424715302612

[20] W. J. Riley, "The calculation of time domain frequency stability," IEEE Ultrasonics, Ferroelectrics and Frequency Control Society. [Online]. Available: https://ieee-uffc.org/frequency-control/educationalresources/the-calculation-of-time-domain-frequency-stability/

[21] S. T. Dawkins, J. J. McFerran, and A. N. Luiten, "Considerations on the measurement of the stability of oscillators with frequency counters," IEEE Transactions on Ultrasonics, Ferroelectrics, and Frequency Control, vol. 54, no. 5, pp. 918-925, May 2007.

\section{APPENDIX A \\ Details of the Frequency Estimation Methods}

The two main non-parametric methods for single tone estimation are Candan estimation [13] and Djukanovic estimation [14]. Prony's method [15], zero crossing method [16] and modified Pisarenko harmonic decomposition method [17] are the commonly used parametric methods.

\section{A. Candan Method}

Ref. [13] discusses the Candan frequency estimation method which is a non-parametric method for single tone estimation. 
A N-point DFT of the signal is obtained and the location $\left(\mathrm{k}_{p}\right)$ of the peak magnitude $\left(R\left[k_{p}\right]\right)$ is obtained for the purpose of coarse estimation. Then, three DFT points corresponding to $\mathrm{k}_{p}$, $\mathrm{k}_{p-1}$ and $\mathrm{k}_{p+1}$ are taken. The normalized estimated frequency is obtained as $\left(\mathrm{k}_{p}+\delta\right) / \mathrm{N}$, where $\delta$ is evaluated from,

$\delta=\frac{\tan ^{-1}\left[\tan \left(\frac{\pi}{N}\right) \operatorname{Re}\left(\frac{R\left[k_{p}-1\right]-R\left[k_{p}+1\right]}{2 R\left[k_{p}\right]-R\left[k_{p}-1\right]-R\left[k_{p}+1\right]}\right)\right]}{\pi / N}$

\section{B. Djukanovic Method}

In this method [14] $\delta$ is obtained from Eq. 4. Three periodogram values $\mathrm{P} 1, \mathrm{P} 2$ and $\mathrm{P} 3$ are obtained at the normalized frequencies, $\theta_{1}=\mathrm{k}_{p} / \mathrm{N}, \theta_{2}=\left(\mathrm{k}_{p}+\delta\right) / \mathrm{N}$ and $\theta_{3}=\left(\mathrm{k}_{p}+2 \delta\right) / \mathrm{N}$, respectively from Eq. 5 .

$$
P(\theta)=\left|\sum_{n=0}^{N-1} x_{n} e^{-j 2 \pi n \theta}\right|
$$

where, $\mathrm{x}_{n}$ is $\mathrm{n}^{\text {th }}$ sampled input. The three points are fitted into a parabolic equation and the abscissa of vertex of the parabola is calculated by Eq. 6, which gives normalized frequency.

$$
\hat{\theta}=\frac{1}{2} \frac{\theta_{3}^{2}\left(P_{1}-P_{2}\right)+\theta_{2}^{2}\left(P_{3}-P_{1}\right)+\theta_{1}^{2}\left(P_{2}-P_{3}\right)}{\theta_{3}\left(P_{1}-P_{2}\right)+\theta_{2}\left(P_{3}-P_{1}\right)+\theta_{1}\left(P_{2}-P_{3}\right)}
$$

\section{Prony's Method}

A computationally simple, single tone estimation through Prony method is presented in [15]. A real valued sampled sinusoid can be written as, $\mathrm{x}_{n}=\mathrm{A} \cos (\omega \mathrm{n}+\Phi), \mathrm{n}=0, \ldots, \mathrm{N}$. Then, the frequency $\omega$ is given by Eq. 7 .

$$
\omega=\cos ^{-1}\left(\left(\sum_{n=2}^{N} x_{n-1}\left(x_{n}+x_{n-2}\right)\right) /\left(2 \sum_{n=2}^{N} x_{n-1}^{2}\right)\right)
$$

\section{Modified Pisarenko Method}

In the modified Pisarenko harmonic decomposition method [17], the frequency of the sinusoid is given by Eq. 8 .

$$
\omega=\cos ^{-1}\left(r_{2}+\sqrt{r_{2}^{2}+8 r_{1}^{2}}\right) /\left(4 r_{1}\right)
$$

In Eq. 8, $r_{1}$ and $r_{2}$ are given by

$$
\begin{aligned}
& r_{1}=\sum_{n=4}^{N-1}\left(x_{n-1}\right)\left[x_{n}+x_{n-2}\right] \\
& r_{2}=\sum_{n=5}^{N}\left(x_{n-2}\right)\left[x_{n}+x_{n-4}\right]
\end{aligned}
$$

\section{E. Zero crossing Interpolation Method}

The frequency of a sinusoidal signal is most commonly measured by counting the zero crossing events in a given time period. The error introduced in the detection technique due to linear interpolation and noise has been analyzed in [16]. 